\title{
Lymphoma Presenting as Sister Mary Joseph's Nodule Sparing Intra-Abdominal Involvement
}

\author{
Ying-Ih Shih, ,a,c Po-Min Chen, ,ac Paul Chih-Hsueh Chen, ${ }^{\mathrm{b}, \mathrm{c}}$ Liang-Tsai Hsiao ${ }^{\mathrm{a}, \mathrm{c}}$ \\ ${ }^{a}$ Division of Medical Oncology, Department of Medicine, Taipei Veterans General Hospital; ${ }^{b}$ Department of Pathology, \\ Taipei Veterans General Hospital; ${ }^{c}$ National Yang-Ming University School of Medicine, Taipei, Taiwan
}

Received January 16, 2006; received in revised form February 10, 2006; accepted February 14, 2006

Int J Hematol. 2006;83:194. doi: 10.1532/IJH97.06015

(C)2006 The Japanese Society of Hematology

A 73-year-old woman received a diagnosis of diffuse large B-cell lymphoma, Ann Arbor stage II, in November 2004, when several enlarged lymph nodes over the left neck and axilla were noted. Because of a poor response to the first-line regimen (cyclophosphamide, vincrinstine, epirubicin, and prednisolone), chemotherapy with the second-line regimen containing rituximab, ifosfamide, cisplatin, and etoposide (R-ICE) and involving field radiation was given between January and May 2005 with a nearly complete response. However, a solitary umbilical mass was found in July 2005. The examination of an abdominal computed tomography (CT) scan did not disclose intra-abdominal or retroperitoneal lesions (Figure 1). In addition, a whole body positron emission tomography (PET) scan only revealed ${ }^{18} \mathrm{~F}$-fluorodeoxyglucose (FDG) uptake in the left axilla, bilateral paratracheal chains, umbilical, and bilateral inguinal regions with no intra-abdominal uptake (Figure 2). The biopsy of the umbilical mass confirmed the lymphoma

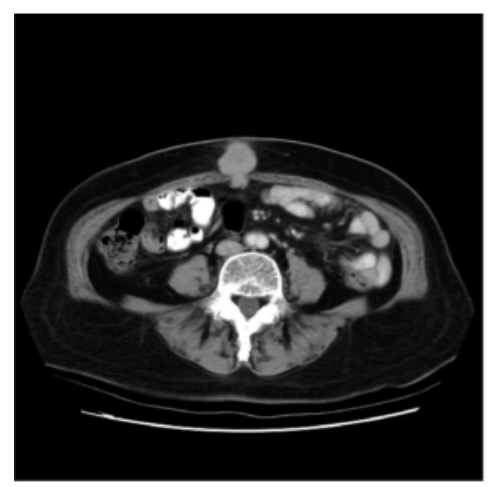

Figure 1. Computed tomography scan of the abdomen. There was 1 soft tissue density at the umbilical area without enlarged lymph nodes in the abdomen.

Correspondence and reprint requests: Liang-Tsai Hsiao, MD, Division of Medical Oncology, Taipei Veterans General Hospital, No. 201, Sec. 2, Shi-Pai Road, Taipei 112, Taiwan; 886-2-28712121 \#2507; fax: 886-2-28732184 (e-mail: hs1085@ms9.hinet.net). involvement (Figure 3). Thereafter, the disease was refractory to subsequent therapy, and the patient died in November 2005.

Umbilical metastasis, also called Sister Mary Joseph's nodule, was thought to be mainly derived from malignancies of the digestive and gynecological systems. Only 5 cases were reported from non-Hodgkin's lymphoma since 1996, and they usually manifested along with intra-abdominal involvements. Our case illustrates additional information about Sister Mary Joseph's nodule as the involvement of lymphoma, mainly that lymphoma cells might spare intra-abdominal involvement.

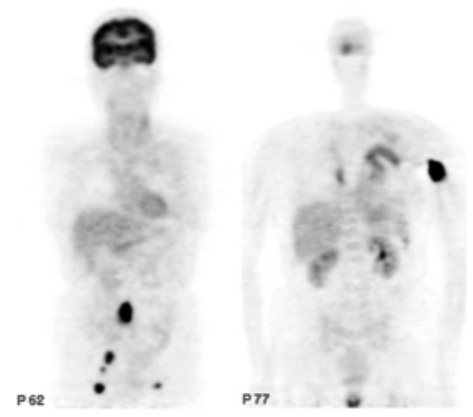

Figure 2. Positron emission tomography scan of the whole body. There were multiple areas with intense ${ }^{18} \mathrm{~F}$-fluorodeoxyglucose uptake on the left axilla, left upper lung bilateral paratracheal chains, and umbilical and bilateral inguinal regions, with no lesions in the abdomen.

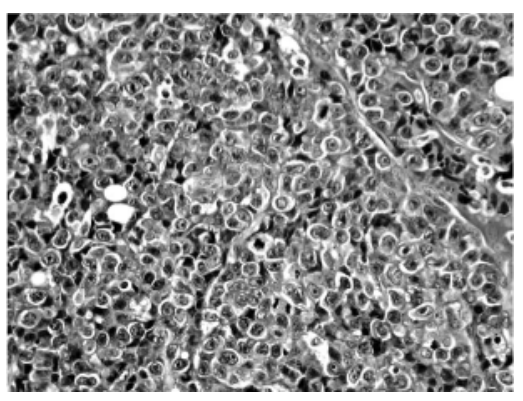

Figure 3. Pathology of umbilical mass biopsy. There was infiltration of diffuse large lymphoid cells that had a high N/C ratio, mitotic figures, and CD20 positive staining. Hematoxylin and eosin stain, original magnification $\times 400$. 